\title{
Families of Integrable Equations ${ }^{\star}$
}

Pavlos KASSOTAKIS ${ }^{\dagger}$ and Maciej NIESZPORSKI $\ddagger$

$\dagger$ Department of Mathematics and Statistics University of Cyprus, P.O. Box: 20537, 1678 Nicosia, Cyprus

E-mail: kassotakis.pavlos@ucy.ac.cy, pavlos1978@gmail.com

$\ddagger$ Katedra Metod Matematycznych Fizyki, Uniwersytet Warszawski, ul. Hoża 74, 00-682 Warszawa, Poland

E-mail: maciejun@fuw.edu.pl

Received May 23, 2011, in final form October 20, 2011; Published online October 28, 2011

http://dx.doi.org/10.3842/SIGMA.2011.100

Abstract. We present a method to obtain families of lattice equations. Specifically we focus on two of such families, which include 3-parameters and their members are connected through Bäcklund transformations. At least one of the members of each family is integrable, hence the whole family inherits some integrability properties.

Key words: integrable lattice equations; Yang-Baxter maps; consistency around the cube

2010 Mathematics Subject Classification: 82B20; 37K35; 39A05

\section{Introduction}

Discrete mathematics returned on the interest of mathematicians at the beginning of the $20^{\text {th }}$ century. Poincaré, Birkhoff, Ritt (1924) [1], Julia, Fatou (1918-1923) [2, 3] and many others saw the necessity of exploring the discrete scene. Unfortunately, this trend was paused through the two big wars and only after 1960, keeping pace with the revolution caused by the discovery of soliton from Zabusky and Kruskal [4], mathematicians started to investigate discrete systems in the context of integrable systems.

It was the work of Hirota [5], as well as Ablowitz et al. [6] and separately Capel and his school [7], which introduced lattice and differential difference analogues of many integrable PDE's. The introduction of discrete versions of integrable ODE's, surprisingly, came later with the QRT family of mappings by Quispel, Roberts and Thomson [8] and by the work of Papageorgiou et al. [9, 10], where Liouville integrable maps [11] were obtained by imposing periodic staircase initial data on integrable lattices. Another way to obtain integrable mappings from an integrable lattice equation was suggested in series of papers $[12,13,14]$. Actually with this procedure one can get involutive mappings (composition of the map with itself is the identity map) which are set theoretical solutions of the quantum Yang-Baxter equation the so called Yang-Baxter maps [15, 16, 12, 17]. As in our previous work [18], we focus here on the inverse procedure, i.e. how to obtain integrable lattice equations from involutive mappings that may or may not satisfy the Yang-Baxter equation.

The main result of the paper is that the procedure we have in mind can lead to families of equations. It is necessary to mention that points of the lattice may not be related in a unique way. The members of families are related by a Bäcklund transformation (see Section 6) and since in considered cases at least one of the members is integrable, the whole family inherits

${ }^{\star}$ This paper is a contribution to the Proceedings of the Conference "Symmetries and Integrability of Difference Equations (SIDE-9)" (June 14-18, 2010, Varna, Bulgaria). The full collection is available at http://www.emis.de/journals/SIGMA/SIDE-9.html 
some properties from the distinguished member. We stress that solutions of each member of the family can be obtained from solutions of the integrable member by discrete quadratures (which can be regarded as sort of Bäcklund transformation) and in this sense each member of the family is integrable. However, we discuss here hallmarks of integrability of the members of the family such as consistency around the cube property or $\tau$-function formulation. Notion of the family of discrete integrable systems should not be confused with notion of hierarchies of integrable systems. The later notion was widely investigated in the literature whereas for the former one we can indicate only the articles that investigate the family of discrete KdV equations [19] and the family of discrete Boussinesq equations [20, 21, 22].

We discuss here two examples, the first one is continuation of our previous paper [18]. We introduce a family of difference equations associated with type III of maps discussed in [12, 13] (we introduced families related to types IV and V in [18]). Example of the map of type III is a map $\mathbb{C}^{2} \ni(u, v) \mapsto(U, V) \in \mathbb{C}^{2}$

$$
U=v \frac{p u-q v}{q u-p v}, \quad V=u \frac{p u-q v}{q u-p v} .
$$

and the three parameter family of equations (see Section 3) reads

$$
\psi_{12}=\psi+a \ln \frac{p u-q v}{q u-p v}+\left(p^{2}-q^{2}\right)\left[b \frac{u v}{q u-p v}-c \frac{1}{p u-q v}\right]
$$

where $u$ and $v$ are given implicitly by

$$
a \ln u+p\left(b u+c \frac{1}{u}\right)=\psi_{1}+\psi, \quad a \ln v+q\left(b v+c \frac{1}{v}\right)=\psi_{2}+\psi
$$

function $\psi$ is dependent variable on $\mathbb{Z}^{2}$ and we denote $\psi(m, n)=: \psi, \psi(m+1, n)=: \psi_{1}, \psi(m, n+$ 1) $=: \psi_{2}, \psi(m+1, n+1)=: \psi_{12}, p:=p(m)$ and $q:=q(n)$ are given functions of a single variable and $a, b$ and $c$ are arbitrary constants (we assume that one of the constants $a, b$ or $c$ is not equal to zero). However, ought to possible branching in formulas (1.2), the system (1.1), (1.2) needs specifying (as it was pointed us by Professors Frank Nijhoff and Yuri Suris). The specification is achieved by demanding that functions $u$ and $v$ obey

$$
u_{2}=v \frac{p u-q v}{q u-p v}, \quad v_{1}=u \frac{p u-q v}{q u-p v} .
$$

After this specification there is still some freedom left in finding solutions of (1.1), (1.2) for given initial conditions on $\psi$. The freedom lies in finding the initial conditions for $u$ and $v$ out of initial conditions on $\psi$ by means of (1.2). The solution need not to be unique.

All the equations within the family are consistent around the cube (for the consistency around the cube property see $[23,24,25,26]$, notice we resign from multiaffinity assumption of paper [25]). We find especially interesting the fact that we obtain examples of lattice equations together with transformations which can be regarded as Bäcklund transformations but not in the usual sense; we usually require Bäcklund transformation to be linearisable (see Definition 5) and this requirement is violated in these examples. Therefore Lax pair could not be easily found from this sort of Bäcklund transformation and it is not clear if the Lax pair exists in these cases. Members of the family are Hirota's sine-Gordon equation (choice of parameters $b=0=c$ ) referred also to as lattice potential modified $\mathrm{KdV}$ [27, 28, 29, 30, 31, 14, 32] (see Section 2 where we discuss various forms of lattice equations)

$$
p\left(x x_{1}+x_{2} x_{12}\right)=q\left(x x_{2}+x_{1} x_{12}\right)
$$


and lattice Schwarzian KdV [28] in a disguise, see Section 2 (choice of parameters $a=0=b$ or $a=0=c)$

$$
p^{2}\left(y_{12}+y_{1}\right)\left(y_{2}+y\right)=q^{2}\left(y_{12}+y_{2}\right)\left(y_{1}+y\right) .
$$

In the second example we go away from the maps of papers $[12,13]$ and consider the map

$$
U=v+k\left(1-\frac{v}{u}\right), \quad V=u+k\left(-1+\frac{u}{v}\right),
$$

which gives also a 3 parameter family of equations (see Section 5) including Hirota's KdV lattice equation [5]

$$
x_{12}-x=\kappa\left(\frac{1}{x_{2}}-\frac{1}{x_{1}}\right)
$$

and two further bilinear equations

$$
y_{1} y-y_{12} y_{1}=\kappa\left(y_{12} y+y_{1} y_{2}\right), \quad z_{12} z+z_{1} z_{2}=z_{12} z_{2}+z_{12} z_{1} .
$$

In this case an interesting fact is that the procedure yields $\tau$-function representation of the family (see e.g. [19])

$$
\tau_{112} \tau-\kappa \tau_{11} \tau_{2}=\tau_{12} \tau_{1}, \quad \tau_{122} \tau+\kappa \tau_{22} \tau_{1}=\tau_{12} \tau_{2}
$$

In Section 2, we give an overview of point transformations, Bäcklund transformations and difference substitutions and touch the issue of equivalence of lattice equations. We proceed in Section 3 where we present the method that leads to families of lattice equations. In Section 4 we relate our findings to some results of the papers [12, 13], followed by Section 5 where we deal with Hirota's KdV lattice equation. Then we explain how to get Bäcklund transformation between members of the families (Section 6) and we end the paper with some conclusions and perspectives for future work.

\section{Point transformations, difference substitutions, Bäcklund transformations and equivalence of lattice equations}

Before we start we would like to give some definitions and recall some well known relations [29, $30,31,19,32]$ between equations that appear in the article (terminology used by various authors is far from being unified). Let us consider $k$ dependent variables of $n$ independent ones: $u^{i}\left(m^{1}, \ldots, m^{n}\right), i=1, \ldots, k$. We denote $M \equiv\left(m^{1}, \ldots, m^{n}\right)$.

Definition 1 (change of independent variables). By change of independent variables we understand the bijection $f: \mathbb{Z}^{n} \rightarrow \mathbb{Z}^{n}$

$$
\tilde{m}^{i}=f^{i}(M), \quad i=1, \ldots, n .
$$

$2 \mathrm{D}$ examples are $\tilde{m}^{1}=m^{1}, \tilde{m}^{2}=m^{1}+m^{2}$, or $\tilde{m}^{1}=m^{1}+2 m^{2}, \tilde{m}^{2}=m^{1}+m^{2}$.

Definition 2 (point transformations not altering independent variables). By point transformation not altering independent variables we understand an invertible map $F$ between subsets of $\mathbb{C}^{k}$

$$
\tilde{u}^{i}(M)=F^{i}\left(u^{1}(M), \ldots, u^{k}(M) ; M\right), \quad i=1, \ldots, k .
$$


Definition 3 (equivalence of lattice equations). Two lattice equations are equivalent if and only if there exists composition of point transformation with change of independent variables which maps solutions of one equation to solutions of the second one.

Examples of various disguises of the same equation are

- Hirota's sine-Gordon equation

$$
q \sin \left(\psi_{12}+\psi-\psi_{1}-\psi_{2}\right)=p \sin \left(\psi_{12}+\psi+\psi_{1}+\psi_{2}\right)
$$

turns into

$$
\left(H 3^{0}\right): \quad p\left(x x_{1}+x_{2} x_{12}\right)=q\left(x x_{2}+x_{1} x_{12}\right)
$$

$H 3^{0}$ equation from ABS list [25] by means of point transformation $x=i^{m+n} e^{2 i(-1)^{n} \psi}$. $H 3^{0}$ in turn can be transformed into lattice potential modified $\mathrm{KdV}$

$$
p\left(w w_{1}-w_{2} w_{12}\right)=q\left(w w_{2}-w_{1} w_{12}\right)
$$

by substitution $x=i^{m+n} w$.

- Schwarzian KdV equation (or cross ratio equation, or equation $Q 1^{0}$ on $\mathrm{ABS}$ list)

$$
\frac{\left(z_{12}-z_{1}\right)\left(z_{2}-z\right)}{\left(z_{12}-z_{2}\right)\left(z_{1}-z\right)}=\frac{q^{2}}{p^{2}}
$$

under the point transformation $z=(-1)^{m+n} y$ turns into

$$
\left(A 1^{0}\right): \quad p^{2}\left(y_{12}+y_{1}\right)\left(y_{2}+y\right)=q^{2}\left(y_{12}+y_{2}\right)\left(y_{1}+y\right)
$$

which in the paper [25] got its own name $A 1^{0}$.

Definition 4 (difference substitutions). Let $j$ points $M^{i}, i=1, \ldots, j$ of a lattice are given. By difference substitution of order $j$ we understand a transformation

$$
\tilde{u}^{i}(M)=F^{i}\left(u^{1}\left(M^{1}\right), \ldots, u^{k}\left(M^{1}\right), \ldots, u^{1}\left(M^{j}\right), \ldots, u^{k}\left(M^{j}\right) ; M\right), \quad i=1, \ldots, k .
$$

Every point transformation is difference substitutions of order 1. Standard examples of difference substitution (of order 2, 3 and 4 respectively) are

- potential relation

$$
v=\frac{1}{\alpha-\beta}\left(u_{2}-u_{1}\right)
$$

between lattice potential $\mathrm{KdV}$

$$
\left(u_{12}-u\right)\left(u_{1}-u_{2}\right)=\alpha^{2}-\beta^{2}
$$

and Hirota's difference KdV

$$
v_{12}-v=\frac{\alpha+\beta}{\alpha-\beta}\left(\frac{1}{v_{1}}-\frac{1}{v_{2}}\right)
$$


- Miura-type transformation

$$
v=\frac{\beta \psi_{2}-\alpha \psi_{1}}{(\beta-\alpha) \psi}
$$

between $H 3^{0}$ (Hirota's sine-Gordon or lattice modified potential KdV)

$$
\alpha\left(\psi_{2} \psi_{12}-\psi \psi_{1}\right)=\beta\left(\psi_{1} \psi_{12}-\psi \psi_{2}\right)
$$

and Hirota's difference KdV;

- and finally the introduction of $\tau$ function

$$
v=\frac{\tau_{12} \tau}{\tau_{1} \tau_{2}}
$$

which transform every solution of the compatible system

$$
\tau_{112} \tau-\kappa \tau_{11} \tau_{2}=\tau_{12} \tau_{1}, \quad \tau_{122} \tau+\kappa \tau_{22} \tau_{1}=\tau_{12} \tau_{2}
$$

to solution of Hirota's difference KdV.

To the end we propose draft definition of Bäcklund transformation which is convenient for our purposes. However we are aware that the definition is not exhaustive (some transformation that deserve this name can be not covered by the definition).

Definition 5 (Bäcklund transformations (in narrow sense)). By Bäcklund transformation we understand here a transformation between two equations $F\left(u_{12}, u_{1}, u_{2}, u\right)=0$ and $\tilde{F}\left(\tilde{u}_{12}, \tilde{u}_{1}, \tilde{u}_{2}, \tilde{u}\right)=0$

$$
\tilde{u}_{1}=f\left(\tilde{u}, u, u_{1}\right), \quad \tilde{u}_{2}=g\left(\tilde{u}, u, u_{2}\right),
$$

which is invertible to

$$
u_{1}=\tilde{f}\left(u, \tilde{u}, \tilde{u}_{1}\right), \quad u_{2}=\tilde{g}\left(u, \tilde{u}, \tilde{u}_{2}\right),
$$

where functions $f$ and $g$ are fractional linear in $\tilde{u}$ and functions $\tilde{f}, \tilde{g}$ are function fractional linear in $u$.

A classical example of Bäcklund transformation between

$$
p\left(x x_{1}+x_{2} x_{12}\right)-q\left(x x_{2}+x_{1} x_{12}\right)=0
$$

and

$$
p^{2}\left(y_{12}+y_{1}\right)\left(y_{2}+y\right)=q^{2}\left(y_{12}+y_{2}\right)\left(y_{1}+y\right)
$$

is the transformation

$$
y_{1}+y=p x_{1} x, \quad y_{2}+y=q x_{2} x .
$$

\section{Outline of the method}

We consider the $\mathbb{Z}^{2}$ lattice together with its horizontal edges (which can be viewed as set of ordered pair of points of $\mathbb{Z}^{2}$, i.e. $\left.E_{h}=\left\{((m, n),(m+1, n)) \mid(m, n) \in \mathbb{Z}^{2}\right\}\right)$ and the vertical ones $\left(E_{v}=\left\{((m, n),(m, n+1)) \mid(m, n) \in \mathbb{Z}^{2}\right\}\right)$. We take into account a function $u$ which is given on horizontal edges $u: E_{h} \rightarrow \mathbb{C}$ and a function $v$ given on vertical ones $v: E_{v} \rightarrow \mathbb{C}$. Shift operators $T_{1}$ and $T_{2}$ act on horizontal edges in standard way $T_{1}((m, n),(m+1, n)):=((m+$ $1, n),(m+2, n)), T_{2}((m, n),(m+1, n)):=((m, n+1),(m+1, n+1))$ (and similarly for vertical edges). We use convention to denote shift action on a function by subscripts $T_{1} u:=u_{1}$.

Now, the outline of the method we developed in [18] can be presented as follows. 


\subsection{From equations to involutive maps. Idea system}

Take a function $x$ given on vertices of the lattice and which obeys $H 3^{0}$ equation

$$
p\left(x x_{1}+x_{2} x_{12}\right)=q\left(x x_{2}+x_{1} x_{12}\right) .
$$

Introduce fields $u$ and $v$ given on horizontal and vertical edges respectively (fields $\mathrm{u}$ and $\mathrm{v}$ are actually the invariants of a symmetry group of the lattice equation (3.1) as it was shown in [13])

$$
u=x x_{1}, \quad v=x x_{2} .
$$

We get

$$
u_{2} u=v_{1} v, \quad p\left(u_{2}+u\right)=q\left(v_{1}+v\right)
$$

and we arrive at the system of equations

$$
u_{2}=v \frac{p u-q v}{q u-p v}, \quad v_{1}=u \frac{p u-q v}{q u-p v} .
$$

The main idea is to investigate system (3.2) rather than equation (3.1) itself. We dare to refer to the system (3.2) as to 2D Idea system III. The point is that the system (3.2) admits, as we shall see, three parameter family of potentials $\psi$ given on vertices of the lattice. Every "potential image" of (3.2) we refer to as idolon (adopting Plato terminology of Ideas and idolons).

First we apply the standard procedure for reinterpretation of equations on a lattice as a map. The reinterpretation is based on identification (see Fig. 1)

$$
u(m, n)=u, \quad v(m, n)=v, \quad u(m, n+1)=U, \quad v(m+1, n)=V,
$$

which turns system (3.2) into $\mathbb{C}^{2} \rightarrow \mathbb{C}^{2}$ map

$$
U=v \frac{p u-q v}{q u-p v}, \quad V=u \frac{p u-q v}{q u-p v} .
$$

We arrive at an involutive Yang-Baxter map that belongs to family of maps denoted by $F_{\text {III }}$ (see [12]).
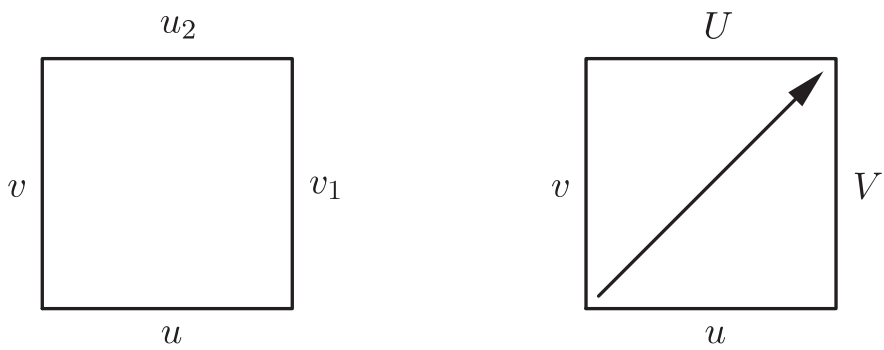

Figure 1. Variables on edges of a $\mathbb{Z}^{2}$ lattice (left picture) and arguments and values of a $\mathbb{C}^{2} \mapsto \mathbb{C}^{2}$ map (right picture).

\subsection{Finding functions such that $F(U)+G(V)=f(u)+g(v)$}

The next step is to find such functions $F$ and $G$ such that for the map (3.4)

$$
F(U)+G(V)=f(u)+g(v) .
$$


holds. Anticipating facts, the functions will allow us to introduce a family of potentials in the next subsection. Differentiation of (3.5) with respect to $u$ and $v$ yields

$$
\begin{aligned}
& -F^{\prime \prime}(U) q U^{2}\left(p U^{2}-2 q U V+p V^{2}\right)+F^{\prime}(U) 2(q U-p V) q U V \\
& \quad+G^{\prime \prime}(V) p V^{2}\left(q U^{2}-2 p U V+q V^{2}\right)+G^{\prime}(V) 2(q U-p V) p U V=0 .
\end{aligned}
$$

The equation above should hold for every value of $U$ and $V$ respectively. The equation has the form

$$
\begin{aligned}
& -p q U^{4} F^{\prime \prime}(U)+2 q^{2} U^{2}\left(U F^{\prime \prime}(U)+F^{\prime}(U)\right) V-p q U\left(U F^{\prime \prime}(U)+F^{\prime}(U)\right) V^{2} \\
& +\alpha(V) U^{2}+\beta(V) U+\gamma(V)=0,
\end{aligned}
$$

so $F(U)$ must satisfy (necessary but not sufficient condition) the ODE

$$
-p q U^{4} F^{\prime \prime}(U)+c_{2} U^{2}+c_{1} U+c_{0}=0 .
$$

with some constants $c_{1}, c_{2}$ and $c_{0}$. Similarly we get

$$
p q V^{4} G^{\prime \prime}(V)+d_{2} V^{2}+d_{1} V+d_{0}=0 .
$$

Checking obtained by this way solutions we obtain

$$
F(U)+G(V)=a \ln (U / V)+b(p U-q V)+c\left(\frac{p}{U}-\frac{q}{V}\right)+d
$$

and we find that for the map (3.4) the following equality holds

$$
a \ln (U / V)+b(p U-q V)+c\left(\frac{p}{U}-\frac{q}{V}\right)=-\left[a \ln (u / v)+b(p u-q v)+c\left(\frac{p}{u}-\frac{q}{v}\right)\right] .
$$

\subsection{Potentials of the Idea systems. Idolons}

Returning to equations on the lattice (by means of (3.3)) one can rewrite (3.6) as

$$
\left(T_{2}+1\right)\left(a \ln u+b p u+c \frac{p}{u}+d\right)=\left(T_{1}+1\right)\left(a \ln v+b q v+c \frac{q}{v}+d\right) .
$$

It means there exists function $\psi$ such that

$$
a \ln u+p\left(b u+c \frac{1}{u}\right)+d=\psi_{1}+\psi, \quad a \ln v+q\left(b v+c \frac{1}{v}\right)+d=\psi_{2}+\psi
$$

where $a, b, c$ and $d$ are arbitrary constants (we assume that one of the constants $a, b, c$ is not equal zero). The constant $d$ can be always removed by redefinition $\psi \rightarrow \psi+\frac{1}{2} d$ and we neglect it

$$
a \ln u+p\left(b u+c \frac{1}{u}\right)=\psi_{1}+\psi, \quad a \ln v+q\left(b v+c \frac{1}{v}\right)=\psi_{2}+\psi
$$

System (3.7) and Idea system (3.2) give rise to

$$
\psi_{12}=\psi+a \ln \frac{p u-q v}{q u-p v}+\left(p^{2}-q^{2}\right)\left[b \frac{u v}{q u-p v}-c \frac{1}{p u-q v}\right]
$$

so we get three parameter family of equations. Note that in general, (3.2) does not follows from (3.7) and (3.9) and therefore we will treat (3.2) as an additional condition that must be satisfied. As we have said in the introduction, choice of parameters $b=0=c$ leads to equation 
$H 3^{0}(2.1)$ whereas choice of parameters either $a=0=b$ or $a=0=c$ leads to equation $A 1^{0}(2.2)$. Every such potential representation of the Idea system we refer to as idolon of the Idea system. To the end let us write another idolon. Namely, $a=0$ yields the equation

$$
\frac{\psi_{2}-\psi_{1}}{\psi_{12}-\psi}=\frac{p^{2}+q^{2}}{p^{2}-q^{2}}-\frac{p q}{p^{2}-q^{2}}\left(\frac{u}{v}+\frac{v}{u}\right)
$$

where $u$ and $v$ are solutions of the following quadratic equations

$$
p\left(b u^{2}+c\right)=\left(\psi_{1}+\psi\right) u, \quad q\left(b v^{2}+c\right)=\left(\psi_{2}+\psi\right) v
$$

and we still assume that (3.2) holds.

\subsection{Extension to multidimension, multidimensional consistency of idolons of $\boldsymbol{I}_{\mathrm{III}}$}

The system (3.2) can be extended to multidimension. We denote by $s^{i}$ (mind superscript!) function given on edges in $i$-th direction of the $\mathbb{Z}^{n}$ lattice, by subscript we denote forward shift in indicated direction. The extension reads

$$
\left(I_{\mathrm{III}}\right): \quad s_{j}^{i}=s^{j} \frac{p^{i} s^{i}-p^{j} s^{j}}{p^{j} s^{i}-p^{i} s^{j}}, \quad i, j=1, \ldots, n, \quad i \neq j,
$$

where $p^{i}$ is given function and can depend only on $i$-th independent variable.

The crucial fact is the system is compatible

$$
s_{j k}^{i}=s_{k j}^{i}
$$

Moreover, we have

$$
\left(T_{j}+1\right)\left[a \ln s^{i}+p^{i}\left(b s^{i}+c \frac{1}{s^{i}}\right)\right]=\left(T_{i}+1\right)\left[a \ln s^{j}+p^{i}\left(b s^{j}+c \frac{1}{s^{i}}\right)\right] .
$$

It means that there exists scalar function $\psi$ such that

$$
a \ln s^{i}+p^{i}\left(b s^{i}+c \frac{1}{s^{i}}\right)=\psi_{i}+\psi, \quad i=1, \ldots, n .
$$

From (3.10) and (3.13) we infer that

$$
\begin{aligned}
& \psi_{i j}=\psi+a \ln \frac{p^{i} s^{i}-p^{j} s^{j}}{p^{j} s^{i}-p^{i} s^{j}}+\left[\left(p^{i}\right)^{2}-\left(p^{j}\right)^{2}\right]\left[b \frac{s^{i} s^{j}}{p^{j} s^{i}-p^{i} s^{j}}-c \frac{1}{p^{i} s^{i}-p^{j} s^{j}}\right] \\
& i, j=1, \ldots, n, \quad i \neq j
\end{aligned}
$$

where $s^{i}$ and $s^{j}$ are given implicitly by means of (3.13). Due to (3.11) the system (3.14) is multidimensionaly consistent (compatible) and we clarify what we mean by that in the following theorem (by i-th initial line we understand in what follows the set $l^{i}=\left\{\left(m_{1}, \ldots, m_{n}\right) \in \mathbb{Z}^{n} \mid \forall k \neq\right.$ $\left.i: m_{k}=0\right\}$ and by set of initial lines we mean $\left.l=l^{1} \cup \cdots \cup l^{n}\right)$

Theorem 1. For arbitrary initial condition on initial lines $\psi(l)$ there exists solution (we do not exclude singularities) $\psi$ of the multidimensional system (3.13), (3.14) that obeys (3.10). 
Proof. Indeed, take arbitrary initial condition on initial lines $\psi(l)$. Then choose a solution $s^{i}\left(l^{i}\right)$ (in general the value of $s^{i}$ is given on the edge between vertices that $\psi$ and $\psi_{i}$ are given on) of the equation

$$
a \ln s^{i}\left(l^{i}\right)+p^{i}\left(b s^{i}\left(l^{i}\right)+c \frac{1}{s^{i}\left(l^{i}\right)}\right)=\psi_{i}\left(l^{i}\right)+\psi\left(l^{i}\right), \quad i=1, \ldots, n
$$

(this is a place when non-uniqueness may enter). We treat $s^{i}(l)$ as initial conditions for the system (3.10). Due to (3.11) the solution $s^{i}$ of (3.10) with initial conditions $s^{i}(l)$ exists (we admit singularities that come from zeroes of $\left.p^{j} s^{i}-p^{i} s^{j}\right)$. Now, due to identity (3.12) there exists function $\psi$ such that (3.13) holds and the value of $\psi$ at the intersection of initial lines is equal to initial condition at the intersection of initial lines. Since $s^{i}$ obeys (3.10) $\psi$ satisfies (3.14) as well. Finally $\psi$ satisfies the assumed arbitrary initial condition since formulas (3.13) at initial lines coincides with (3.15).

We refer to the system (3.10) as to n-dimensional Idea system III and that is why we have denoted it by $I_{\mathrm{III}}$.

\section{Maps}

As we have already mentioned our inspiration was a survey on Yang-Baxter maps. Our goal now is to relate our findings to some results of the papers $[12,13]$ and justify why it makes sense to talk about the Idea systems

$$
s_{j}^{i}=s^{j} \frac{p^{i} s^{i}-p^{j} s^{j}}{p^{j} s^{i}-p^{i} s^{j}}, \quad i=1, \ldots, n
$$

associated with maps of type III rather than single Idea system. The Idea systems are related by point transformation.

Indeed, first we perform a cosmetic point transformation $s^{i}=p^{i} v^{i}, p^{i^{2}} \rightarrow p^{i}$ and we get

$$
v_{j}^{i}=\frac{v^{j}}{p^{i}} \frac{p^{i} v^{i}-p^{j} v^{j}}{v^{i}-v^{j}},
$$

which in two-dimensional case after identification analogous to the one showed on the Fig. 1 yields $F_{\text {III }}$ map of paper [12]

$$
\left(F_{\mathrm{III}}\right): \quad U=\frac{v}{p} \frac{p u-q v}{u-v}, \quad V=\frac{u}{q} \frac{p u-q v}{u-v} .
$$

In fact by $F_{\text {III }}$ we understand equivalence class of Yang-Baxter maps (cf. [13]) the equations (4.1) and (4.2) belongs to.

Now after the point transformation $v^{i}=u^{i}(-1)^{m_{1}+\cdots+m_{n}}$ we get

$$
u_{j}^{i}=-\frac{u^{j}}{p^{i}} \frac{p^{i} u^{i}-p^{j} u^{j}}{u^{i}-u^{j}}
$$

associated 2D map of which is

$$
\left(c H_{\mathrm{III}}^{A}\right): \quad U=-\frac{v}{p} \frac{p u-q v}{u-v}, \quad V=-\frac{u}{q} \frac{p u-q v}{u-v} .
$$

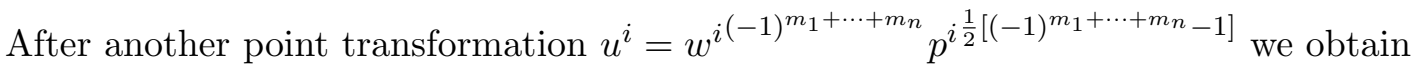

$$
w_{j}^{i}=-\frac{1}{w^{j}} \frac{w^{i}-w^{j}}{p^{i} w^{i}-p^{j} w^{j}}
$$


and its associated map

$$
\left(c H_{\mathrm{III}}^{B}\right): \quad U=-\frac{1}{v} \frac{u-v}{p u-q v}, \quad V=-\frac{1}{u} \frac{u-v}{p u-q v} .
$$

Maps (4.3) and (4.4) are not Yang-Baxter maps but they are companions (if $f:(u, v) \mapsto(U, V)$ is involutive map then the map $(u, V) \mapsto(U, v)$ we refer to as companion of map $f$, cf. [12]) of YangBaxter maps $H_{\mathrm{III}}^{A}, H_{\mathrm{III}}^{B}$ of paper [17]. The maps $H_{\mathrm{III}}^{A}, H_{\mathrm{III}}^{B}$ can be obtained in two-dimensional case by the point transformation $u^{1}=x, u^{2}=-y$ and $w^{1}=x, w^{2}=-\frac{1}{q y}$ respectively

$$
x_{2}=\frac{y}{p} \frac{p x+q y}{x+y}, \quad y_{1}=\frac{x}{q} \frac{p x+q y}{x+y} \quad \text { and } \quad x_{2}=y \frac{q x y+1}{p x y+1}, \quad y_{1}=x \frac{p x y+1}{q x y+1}
$$

and then by mentioned identification (see Fig. 1)

$$
\begin{aligned}
& \left(H_{\mathrm{III}}^{A}\right): \quad U=\frac{v}{p} \frac{p u-q v}{u-v}, \quad V=\frac{u}{q} \frac{p u-q v}{u-v}, \\
& \left(H_{\mathrm{III}}^{B}\right): \quad U=v \frac{q u v+1}{p u v+1}, \quad V=u \frac{q u v+1}{p u v+1} .
\end{aligned}
$$

Idea systems $\left(H_{\mathrm{III}}^{A}\right)$ and $\left(H_{\mathrm{III}}^{B}\right)$ cannot be extended to multidimension (in the sense of [25]).

\begin{tabular}{|c|c|c|}
\hline Type of the map & Example of the map & Identities \\
\hline$F_{\text {III }}$ & $\begin{aligned} U & =\frac{v}{p} \frac{p u-q v}{u-v} \\
V & =\frac{u}{q} \frac{p u-q v}{u-v}\end{aligned}$ & $\begin{array}{l}\frac{U}{V}=\frac{q v}{p u} \\
p U-q V=-(p u-q v) \\
\frac{1}{U}-\frac{1}{V}=-\left(\frac{1}{u}-\frac{1}{v}\right)\end{array}$ \\
\hline$c H_{\mathrm{III}}^{A}$ & $\begin{aligned} U & =-\frac{v}{p} \frac{p u-q v}{u-v} \\
V & =-\frac{u}{q} \frac{p u-q v}{u-v}\end{aligned}$ & $\begin{array}{l}\frac{U}{V}=\frac{q v}{p u} \\
p U-q V=p u-q v \\
\frac{1}{U}-\frac{1}{V}=\frac{1}{u}-\frac{1}{v}\end{array}$ \\
\hline$c H_{\mathrm{III}}^{B}$ & $\begin{aligned} U & =\frac{1}{v} \frac{u-v}{q v-p u} \\
V & =\frac{1}{u} \frac{u-v}{q v-p u}\end{aligned}$ & $\begin{array}{l}\frac{U}{V}=\frac{u}{v} \\
p U+\frac{1}{U}-q V-\frac{1}{V}=p u+\frac{1}{u}-q v-\frac{1}{v} \\
p U-\frac{1}{U}-q V+\frac{1}{V}=-\left(p u-\frac{1}{u}-q v+\frac{1}{v}\right)\end{array}$ \\
\hline$H_{\mathrm{III}}^{A}$ & $\begin{array}{l}U=\frac{v(p u+q v)}{p(u+v)} \\
V=\frac{u(p u+q v)}{q(u+v)}\end{array}$ & $\begin{array}{l}\frac{U}{V}=\frac{q v}{p u} \\
p U+q V=p u+q v \\
\frac{1}{U}+\frac{1}{V}=\frac{1}{u}+\frac{1}{v}\end{array}$ \\
\hline$H_{\mathrm{III}}^{B}$ & $\begin{array}{l}U=v \frac{q u v+1}{p u v+1} \\
V=u \frac{p u v+1}{q u v+1}\end{array}$ & $\begin{array}{l}U V=u v \\
p U+q V+\frac{1}{U}+\frac{1}{V}=p u+q v+\frac{1}{u}+\frac{1}{v} \\
p U-q V-\frac{1}{U}+\frac{1}{V}=-\left(p u-q v-\frac{1}{u}+\frac{1}{v}\right)\end{array}$ \\
\hline
\end{tabular}

Finally, we list in the Table 1 basic identities of the maps that leads to existence of potentials of the Idea systems to illustrate how the basis changes when one changes a map.

Table 1. Basic identities of the maps that leads to existence of potentials of the Idea system. 


\section{$5 \quad$ Hirota's KdV lattice equation}

As the second example we consider Hirota's KdV lattice equation [5]

$$
x_{12}-x=\kappa\left(\frac{1}{x_{2}}-\frac{1}{x_{1}}\right) .
$$

By the substitution $u=x_{1} x, v=x_{2} x$, we get

$$
u_{2}=v+\kappa\left(1-\frac{v}{u}\right), \quad v_{1}=u+\kappa\left(-1+\frac{u}{v}\right) .
$$

On applying identification (3.3)

$$
u=u(m, n), \quad v=v(m, n), \quad U=u(m, n+1), \quad V=v(m+1, n)
$$

we obtain an involutive mapping associated to system (5.1)

$$
U=v+\kappa\left(1-\frac{v}{u}\right), \quad V=u+\kappa\left(-1+\frac{u}{v}\right) .
$$

Mapping (5.3) satisfies (this is the outcome of searching for such functions $F$ and $G$ that $F(U)+$ $G(V)=f(u)+g(v)$ as described in the previous section):

$$
\frac{U}{V}=\frac{v}{u}, \quad(U-\kappa)(V+\kappa)=(u-\kappa)(v+\kappa), \quad \frac{V(U-\kappa)}{U(V+\kappa)}=\frac{v(u-\kappa)}{u(v+\kappa)},
$$

hence (coming back to lattice variables (5.2)) we can introduce the potentials $x, y$ and $z$

$$
\begin{array}{ll}
u=x_{1} x, & v=x_{2} x, \\
u-\kappa=y_{1} / y, & v+\kappa=y / y_{2}, \\
\frac{u-\kappa}{u}=z_{1} / z, & \frac{v+\kappa}{v}=z_{2} / z .
\end{array}
$$

Eliminating $u$ and $v$ from (5.1) we arrive at the following lattice equations

$$
\begin{aligned}
& x_{12}-x=\kappa\left(1 / x_{2}-1 / x_{1}\right), \quad y_{1} y-y_{12} y_{1}=\kappa\left(y_{12} y+y_{1} y_{2}\right), \\
& z_{12} z+z_{1} z_{2}=z_{12} z_{2}+z_{12} z_{1} .
\end{aligned}
$$

One can treat the equations as representatives of a three-parameter family of equations on $\phi$

$$
\begin{aligned}
& \frac{\phi_{12} \phi}{\phi_{1} \phi_{2}}=\left[(u-\kappa)(v+\kappa)+\kappa^{2}\right]^{a(-1)^{m+n+1}-b} u^{b-c} v^{b+c}, \\
& \frac{\phi_{1}}{\phi}=u^{a(-1)^{m+n}-b}(u-\kappa)^{b+c}, \quad \frac{\phi_{2}}{\phi}=v^{a(-1)^{m+n}-b}(v+\kappa)^{b-c},
\end{aligned}
$$

corresponding to the choice of parameters $b=0=c, a=0=b$ and $a=0=c$ respectively.

What more important is that from (5.4) we infer

$$
\frac{z_{1}}{z}=\frac{y_{1}}{x_{1} x y}, \quad \frac{z_{2}}{z}=\frac{y}{y_{2} x_{2} x} .
$$

Compatibility condition that guarantees existence of function $z$ reads

$$
\left(\frac{x_{2}}{x_{1}}\right)^{2}=\left(\frac{y_{12} y}{y_{1} y_{2}}\right)^{2}
$$

from where we get

$$
x=\frac{\tau_{12} \tau}{\tau_{1} \tau_{2}}, \quad y=\frac{\tau_{2}}{\tau_{1}}, \quad z=\frac{\tau}{\tau_{12}} .
$$

Eliminating $x, y$ and $z$ from (5.4) we arrive at a compatible pair of bilinear forms of Hirota's $\mathrm{KdV}$ (cf. [19])

$$
\tau_{112} \tau-\kappa \tau_{11} \tau_{2}=\tau_{12} \tau_{1}, \quad \tau_{122} \tau+\kappa \tau_{22} \tau_{1}=\tau_{12} \tau_{2} .
$$




\section{Bäcklund transformations between idolons}

In both presented examples one can find Bäcklund transformation between idolons. For instance eliminating $u$ and $v$ from first two lines of (5.4) one gets Bäcklund transformation between first two equations of (5.5)

$$
\frac{y_{1}}{y}=x_{1} x-k, \quad \frac{y}{y_{2}}=x_{2} x+k .
$$

Similarly in the case of $I_{\mathrm{III}}$ one can obtain Bäcklund transformation (2.3).

Finally, we present the Bäcklund transformation between $A 1^{0}(2.2)$ and the idolon (3.8), (3.9). Namely, if $y$ satisfies $A 1^{0}$ then

- function $\psi$ given by

$$
\begin{aligned}
& a \ln \frac{p}{y_{1}+y}+\frac{b p^{2}}{y_{1}+y}+c\left(y_{1}+y\right)=\psi_{1}+\psi, \\
& a \ln \frac{q}{y_{2}+y}+\frac{b q^{2}}{y_{2}+y}+c\left(y_{2}+y\right)=\psi_{2}+\psi
\end{aligned}
$$

exists (compatibility conditions are satisfied due to the fact that $y$ satisfies $A 1^{0}$ );

- functions $u$ and $v$ given by $u=\frac{p}{y_{1}+y}, v=\frac{q}{y_{2}+y}$ obey Idea system (3.2);

- function $\psi$ obeys (3.8), (3.9).

\section{Conclusions}

In this paper we focused on two 3-parameter families of lattice equations. The first one (1.1), (1.2) and (1.3) is related to mappings of type III which were introduced in [12, 13]. Two members (idolons) of the later are, the Hirota's sine-Gordon equation and the lattice Schwarzian KdV [28] in a disguise. Generally, all idolons are connected through Bäcklund transformations and they are 3D-consistent in the sense described in the paper. In the not-too-distant future we are going to investigate families of equations related to given integrable systems not only by discrete quadratures but also by Bäcklund transformation from the Definition 5 .

The second family described by (5.6) and (5.1) is not 3D-consistent. Nevertheless, all of its idolons are connected through Bäcklund transformations, and since an idolon of this family is the Hirota's KdV equation, the whole family inherits some integrability properties e.g. $\tau$-function formulation.

We would like to emphasize once more that the main object under consideration are Idea systems (3.2) (or its $n$-dimensional version (3.10)) and (5.1). The main observation is that the Idea systems admit three-dimensional vector space of scalar potentials (formulas (3.8) in case of two-dimensional Idea $I_{\mathrm{III}}$ and (3.13) in the n-dimensional case, see also second and third formulas of (5.6)). In a forthcoming paper we will discuss all Idea systems that arise from equations of Adler-Bobenko-Suris list. In other words, we plan to investigate all mappings in [12, 13], determine their associate Idea systems and put more light into integrability properties of the associated family of lattice equations. Also, it will be interesting to investigate the mappings that arise when one imposes periodic staircase initial data on these families of lattice equations. Another objective is to derive the discrete Painlevé equations associated with these families.

Finally, we will discuss the case of real-valued functions, which can lead to standard 3Dconsistent lattice equations. For instance for the idolon we proposed in [18]

$$
f_{12}=f+(p-q)\left[v-u+\frac{f_{1}-f_{2}}{(u-v)^{2}}+\frac{(p-q)^{2}}{(u-v)^{3}}\right]
$$




$$
u^{3}+a u=f_{1}-f, \quad v^{3}+b v=f_{2}-f, \quad a-b=3(q-p) .
$$

assuming $f: \mathbb{Z}^{2} \rightarrow \mathbb{R}$ and $a, b>0$ the only real solutions of the cubic equations are

$$
\begin{aligned}
& u=\sqrt[3]{\frac{f_{1}-f}{2}+\sqrt{\frac{\left(f_{1}-f\right)^{2}}{4}+\frac{a^{3}}{27}}}+\sqrt[3]{\frac{f_{1}-f}{2}-\sqrt{\frac{\left(f_{1}-f\right)^{2}}{4}+\frac{a^{3}}{27}}}, \\
& v=\sqrt[3]{\frac{f_{2}-f}{2}+\sqrt{\frac{\left(f_{2}-f\right)^{2}}{4}+\frac{b^{3}}{27}}}+\sqrt[3]{\frac{f_{2}-f}{2}-\sqrt{\frac{\left(f_{2}-f\right)^{2}}{4}+\frac{b^{3}}{27}}} .
\end{aligned}
$$

Then the real lattice equation (7.1), with $u$ and $v$ given by (7.2), is 3D-consistent.

From another perspective, instead of dealing with the family of lattice equations, it seems more fundamental to define a model that consists of the Idea system and the associate potential equation (e.g. equations (3.2), (3.7) or (3.10), (3.13) for the multidimensional extension). Then the family of 3D-consistent (see Theorem 1) lattice equations follows naturally. But what more important, this is a new lattice model, defined in both vertices and edges of a $2 \mathrm{D}$ square lattice ( $n \mathrm{D}$ lattice in the multidimensional extension). Such models have also been introduced in the recent work of Hietarinta and Viallet [33].

\section{Acknowledgements}

We would like to thank organizers of SIDE-9 conference in Varna for their hospitality and financial support. Special thanks to Georgi Grahovski for showing us the other side of Varna. M.N. thanks Frank Nijhoff for pointing papers [20, 21].

\section{References}

[1] Ritt J., Permutable rational functions, Trans. Amer. Math. Soc. 25 (1923), 399-448.

[2] Julia G., Mémoire sur la permutabilité des fractions rationelles, Ann. Sci. Ecole Norm. Sup. (3) 39 (1922), $131-152$.

[3] Fatou P., Sur l'itération analytique et les substitutions pennutables, J. Math. Pure Appl. 23 (1924), 1-49.

[4] Zabusky N.J., Kruskal M.D., Interaction of "solitons" in a collisionless plasma and the recurrence of initial states, Phys. Rev. Lett. 15 (1965), 240-243.

[5] Hirota R., Nonlinear partial difference equation. I. A difference analogue of the Korteweg-de Vries equation, J. Phys. Soc. Japan 43 (1977), 1424-1433.

[6] Ablowitz M.J., Ladik F.J., A nonlinear difference scheme and inverse scattering, Stud. Appl. Math. 55 (1976), 213-229.

[7] Nijhoff F.W., Quispel G.R.W., Capel H.W., Direct linearization of nonlinear difference-difference equations, Phys. Lett. A 97 (1983), 125-128.

[8] Quispel G.R.W., Roberts J.A.G., Thompson C.J., Integrable mappings and soliton equations, Phys. Lett. A 126 (1988), 419-421.

[9] Papageorgiou V.G., Nijhoff F.W., Capel H.W., Integrable mappings and nonlinear integrable lattice equations, Phys. Lett. A 147 (1990), 106-114.

[10] Nijhoff F.W., Papageorgiou V.G., Capel H.W., Quispel G.R.W., The lattice Gel'fand-Dikii hierarchy, Inverse Problems 8 (1992), 597-621.

[11] Veselov A.P., Integrable maps, Russ. Math. Surv. 46 (1991), no. 5, 1-51.

[12] Adler V.E., Bobenko A.I., Suris Yu.B., Geometry of Yang-Baxter maps: pencils of conics and quadrirational mappings, Comm. Anal. Geom. 12 (2004), 967-1007, math.QA/0307009.

[13] Papageorgiou V.G., Tongas A.G., Veselov A.P., Yang-Baxter maps and symmetries of integrable equations on quad-graphs, J. Math. Phys. 47 (2006), 083502, 16 pages.

[14] Bobenko A.I., Suris Yu.B., Discrete differential geometry. Integrable structure, Graduate Studies in Mathematics, Vol. 98, American Mathematical Society, Providence, RI, 2008. 
[15] Drinfeld V.G., On some unsolved problems in quantum group theory, in Quantum Groups, Lecture Notes in Math., Vol. 1510, Springer, Berlin, 1992, 1-8.

[16] Veselov A.P., Yang-Baxter maps and integrable dynamics, Phys. Lett. A 314 (2003), 214-221, math.QA/0205335.

[17] Papageorgiou V.G., Suris Yu.B., Tongas A.G., Veselov A.P., On quadrirational Yang-Baxter maps, SIGMA 6 (2010), 033, 9 pages, arXiv:0911.2895.

[18] Kassotakis P., Nieszporski M., On non-multiaffine consistent around the cube lattice equations, arXiv:1106.0636.

[19] Nijhoff F.W., Ramani A., Grammaticos B., Ohta Y., On discrete Painlevé equations associated with the lattice KdV systems and the Painlevé VI equation, Stud. Appl. Math. 106 (2001), 261-314, solv-int/9812011.

[20] Nijhoff F.W., On some "Schwarzian" equations and their discrete analogues, in Algebraic Aspects of Integrable Systems: In Memory of Irene Dorfman, Editors A.S. Fokas and I.M. Gel'fand, Progr. Nonlinear Differential Equations Appl., Vol. 26, Birkhäuser Boston, Boston, MA, 1997, 237-260.

[21] Nijhoff F.W., Discrete Painlevé equations and symmetry reduction on the lattice, in Discrete Integrable Geometry and Physics (Vienna, 1996), Editors A.I. Bobenko and R. Seiler, Oxford Lecture Ser. Math. Appl., Vol. 16, Oxford Univ. Press, New York, 1999, 209-234.

[22] Nijhoff F.W., A higher-rank version of the Q3 equation, arXiv:1104.1166.

[23] Nijhoff F.W., Walker A.J., The discrete and continuous Painlevé VI hierarchy and the Garnier systems, Glasgow Math. J. 43A (2001), 109-123, nlin.SI/0001054.

[24] Nijhoff F.W., Lax pair for the Adler (lattice Krichever-Novikov) system, Phys. Lett. A 297 (2002), 49-58, nlin.SI/0110027.

[25] Adler V.E., Bobenko A.I., Suris Yu.B., Classification of integrable equations on quad-graphs. The consistency approach, Comm. Math. Phys. 233 (2003), 513-543, nlin.SI/0202024.

[26] Boll R., Classification of 3D consistent quad-equations, arXiv:1009.4007.

[27] Hirota R., Nonlinear partial difference equations. III. Discrete sine-Gordon equation, J. Phys. Soc. Japan 43 (1977), 2079-2086.

[28] Nijhoff F., Capel H., The discrete Korteweg-de Vries equation, Acta Appl. Math. 39 (1995), $133-158$.

[29] Faddeev L., Volkov A.Yu., Hirota equation as an example of an integrable symplectic map, Lett. Math. Phys. 32 (1994), 125-135, hep-th/9405087.

[30] Bobenko A., Pinkall U., Discrete surfaces with constant negative Gaussian curvature and the Hirota equation, J. Differential Geom. 43 (1996), 527-611.

[31] Zabrodin A., Tau-function for discrete sine-Gordon equation and quantum $R$-matrix, solv-int/9810003.

[32] Grammaticos B., Ramani A., Scimiterna C., Willox R., Miura transformations and the various guises of integrable lattice equations, J. Phys. A: Math. Theor. 44 (2011), 152004, 9 pages.

[33] Hietarinta J., Viallet C., Integrable lattice equations with vertex and bond variables, J. Phys. A: Math. Theor. 44 (2011), 385201, 13 pages, arXiv:1105.4996. 